\title{
THE ESTIMATION OF PRECISIONS IN THE PLANNING OF UAS PHOTOGRAMMETRIC SURVEYS
}

\author{
D. Passoni*, B. Federici, I. Ferrando, S. Gagliolo, D. Sguerso \\ Università degli Studi di Genova, DICCA - Laboratory of Geodesy, Geomatics and GIS, Via Montallegro 1, 16145 Genoa \\ daniele.passoni@dicca.unige.it, (sara.gagliolo, ilaria.ferrando)@edu.unige.it, (bianca.federici, domenico.sguerso)@unige.it
}

\section{Commission II, ICWG I/II}

KEY WORDS: UAS photogrammetry, planning survey, expected precision

\begin{abstract}
:
The Unmanned Aerial System (UAS) is widely used in the photogrammetric surveys both of structures and of small areas. Geomatics focuses the attention on the metric quality of the final products of the survey, creating several 3D modelling applications from UAS images. As widely known, the quality of results derives from the quality of images acquisition phase, which needs an a priori estimation of the expected precisions. The planning phase is typically managed using dedicated tools, adapted from the traditional aerial-photogrammetric flight plan. But UAS flight has features completely different from the traditional one. Hence, the use of UAS for photogrammetric applications today requires a growth in knowledge in planning.

The basic idea of this research is to provide a drone photogrammetric flight planning tools considering the required metric precisions, given a priori the classical parameters of a photogrammetric planning: flight altitude, overlaps and geometric parameters of the camera. The created "office suite" allows a realistic planning of a photogrammetric survey, starting from an approximate knowledge of the Digital Surface Model (DSM), and the effective attitude parameters, changing along the route. The planning products are the overlapping of the images, the Ground Sample Distance (GSD) and the precision on each pixel taking into account the real geometry. The different tested procedures, the obtained results and the solution proposed for the a priori estimates of the precisions in the particular case of UAS surveys are here reported.
\end{abstract}

\section{INTRODUCTION}

The development of Unmanned Aerial System (UAS) had a rapid evolution during the last decade, mainly connected to the "emotional" video recordings field, which privileges the radiometric quality of the images, disregarding the analysis of metric precision. The UAS is widely used also in the photogrammetric surveys of structures and small areas, usually extended for a few hectares. Geomatics focuses the attention on the metric quality of the final products of the survey, for creating several 3D modelling applications from UAS images. As widely known, the quality of results strictly depends on the quality of images reached in the acquisition phase, which needs an accurate a priori estimation of the expected precisions of the survey to evaluate the final product.

This topic has been widely studied and discussed for the aerial photogrammetry field and for the close-range terrestrial surveys, but not yet analysed for those carried out by UAS.

In the majority of the cases, the planning phase is typically managed using dedicated tools, which adapt the traditional aerial-photogrammetric flight plan. However, UAS flight features are completely different from the traditional ones: the traditional aerial-photogrammetric survey is characterized by great stability, while the UAS survey permits complex flight plans. The arrangement parameters of a UAS flight can be extremely variable; thus, sometimes the application of traditional aerial-photogrammetric rules for the evaluation of the real achievable precisions is meaningless.

The present work consists in the realization of a suite of tools that can provide adequate planning, analysis and manage for a drone photogrammetric flight. The basic idea is to provide flight planning tools accounting for the required metric precisions, the Ground Sample Distance (GSD), i.e. the dimension of the image pixel projected on the surface, and the number of observations (occurrence). The created "office suite" allows a realistic planning of a photogrammetric survey, starting from an approximate knowledge of the Digital Surface Model (DSM), and the effective attitude parameters, changing along the route. The approximate DSM could be obtained by previous surveys or by a preliminary fast survey at a high distance. The planning products are the overlapping of the images, the GSD and the precision on each pixel, taking into account the real geometry.

\section{THE APPROACHES FOR THE ESTIMATION OF A PRIORI PRECISION IN PHOTOGRAMMETRY}

The purpose of a photogrammetric survey is the metric reconstruction of an object or of a territory, thus, the planning of the survey must provide an evaluation of the expected accuracies. To pursue this goal, the tested a priori estimation methods are: (1) the model of classical aerial photogrammetry proposed by Kraus (1982), (2) the model proposed by Fraser (1992) for the terrestrial surveys of close-range photogrammetry, and (3) the approach suggested in the present work.

\subsection{The Kraus model of classical photogrammetry}

The first approach for the estimation of the a priori precision starts from the classical case of aerial photogrammetry. In particular, Kraus proposes an estimation of the expected accuracies in the traditional case of double-photo restitution. This classical case supposes that the camera axes are parallel and normal to the base joining the cameras. This condition is extremely difficult to achieve with aerial photographs, though an approximatively similar condition can be reached. In aerial 
photogrammetry from UAS, this condition is even more difficult to achieve, due to the increasing difficulties in managing and controlling the vehicle during the flight.

Made this premise, we can compute the object coordinates (X, $\mathrm{Y}, \mathrm{Z}$ ) from the quantities (coordinate image $\xi_{1}$, coordinate image $\eta_{1}$, parallax $p_{\xi}$ ) measured on the image. Then, it is possible to evaluate the accuracy of these indirectly derived coordinates. Supposing the principal distance or focal length $\mathrm{c}$ and the base-length $\mathrm{B}$ error-free, the root mean square error $\sigma_{z}$ will result:

$$
\sigma_{z=} \frac{Z^{2}}{c B} \sigma_{p_{\xi}}
$$

where $\mathrm{Z}$ is the relative flight altitude and $\sigma_{p_{\xi}}$ is the precision of the measurements on the images. In the following Figure 1, the procedure to evaluate the Kraus standard deviation is shown.



Figure 1. Kraus standard deviation evaluations

The Kraus formula represents the standard deviation only along the $\mathrm{Z}$ axis, because is generally the most significant one. Instead, the suite planning tools proposed by the authors evaluate also the planimetric standard deviations.

\subsection{The terrestrial close-range model}

The relations used for terrestrial close-range photogrammetry significantly change, due of the high level of overlapping and the presence of oblique images. In fact, the number of images and their convergence are fundamental aspects to be taken into account for the computation of the final accuracy. According to Fraser (1992), the precision of an object point X, Y, Z can be expressed as:

$$
\sigma_{x y z}=\frac{q d}{c \sqrt{n}} \sigma_{p_{x}}
$$

where $\mathrm{d}$ is the distance between the object and the camera, $\mathrm{c}$ is the focal length, $\mathrm{n}$ is the number of overlapping images, $\mathrm{q}$ is a form factor and $\sigma_{p_{x}}$ is the precision of the measurements on the images. The latter parameter $\left(\sigma_{p_{x}}\right)$ strongly depends by the measurement principle used, but in case of calibrated cameras, it can be assumed equal to the pixel size. The form coefficient $\mathrm{q}$ can be considered equal to 3.5 in case of nadiral acquisition with a standard overlapping equal to $60 \%$ of the image size. It decreases to 3 in case of acquisition with high cross overlapping and it reaches value of 0.4 in case of high convergent geometry.

\subsection{The proposed rigorous approach}

As already mentioned, it is not always possible to estimate the precisions of observation of photogrammetric blocks acquired by UAS using the traditional rules that can be derived by applying the covariance propagation law to the classical geometry case. A more rigorous approach is needed.

Basically, the photogrammetric reconstruction of a points cloud describing an object or a territory is an optical triangulation technique. The fundamental observables are the angles: the measurement of an image coordinate pair $(\xi, \eta)$ on a photograph yields two orthogonal angle observations; in the same way, such directions are the basic observables of a topographic survey with a theodolite. The linear functional and stochastic model of an Aerial Triangulation (AT) can be written as:

$$
\begin{aligned}
& v=A x-l \\
& C_{l}=\sigma_{0}^{2} P^{-1}
\end{aligned}
$$

where $\mathrm{l}, \mathrm{v}$, and $\mathrm{x}$ are the vectors of observations, residuals, and unknown parameters, respectively; $A$ is the design matrix; $C_{l}$ is the covariance matrix of observations; $\mathrm{P}$ is the weight matrix; and $\sigma_{0}$ is the variance factor. From the least squares theory, the covariance matrix of the unknown parameters $C_{x x}$ can be written as:

$$
C_{x x}=\sigma_{0}^{2}\left(A^{T} P A\right)^{-1}
$$

The trace of $\mathrm{C}_{\mathrm{xx}}$ matrix provides the variance, and, consequently, the precision, for the $(\mathrm{X}, \mathrm{Y}, \mathrm{Z})$ coordinates of the object points. Assuming to know the Internal Orientation (IO) of the camera and using the External Orientation (EO) of planning, we can simulate this network and know the standard deviation for each point inside the survey area, provided that it is visible on at least a couple of images. This case supposes that the precision of the measurements on the images $\left(\sigma_{p_{\xi}}\right)$ is equal to one pixel and the a posteriori sigma $0\left(\hat{\sigma}_{0}\right)$ is equal to $1 / 3$ of ground pixel dimensions (GSD).

\section{REALISTIC PLANNING PROCEDURE}

The goal is to realize an "office suite", in Matlab ${ }^{\circledR}$ environment, to plan (pre-survey) and to check (post-survey) the geometric quality of the survey itself.

In the pre-survey phase, the "office suite" permits to design a "realistic" survey planning of the navigation route in respect of the a priori DSM, both providing standard solutions and predicting the expected precisions.

The starting points are: (1) the a priori information about expected precisions and/or ground pixel dimensions (GSD), (2) the overlapping of the projected images on the ground/object (in the two directions, longitudinal and transversal on the UAS navigation route) and/or the number of observations (occurrence) of any point/pixel, (3) an approximate knowledge of the DSM, and (4) the desired attitude parameters changing in the route. The approximate DSM could be obtained by previous surveys or by a preliminary fast survey at a high distance.

The planning products are the overlapping of the images, the GSD and the precision on each pixel, taking into account the effective observed geometry.

In order to achieve these results, the procedure needs the following steps: 
1. choice and selection of the survey area, using Google Maps as background);

2. project parameters definition: type of camera used (IO), overlappings (longitudinal and transversal), flight altitude;

3. computation of the projection centers positions and attitudes;

4. verification of the project parameters using the provided a priori DSM;

5. estimation of the a priori precision for all ground pixels, after a check on visibility of the points on the images.

The estimation of the expected accuracies is performed within this procedure using all the three methods described in Section 2 (Kraus model of classical photogrammetry, terrestrial closerange model photogrammetry and the proposed rigorous approach), providing different results.

\subsection{An example of planning}

In order to explain better the different results obtained with the different approach, an example of planning relative to the survey of the Vernazzola beach (Genova, Italy) is shown. The Vernazzola beach is taken as example because is a flat territory surrounded by buildings, which constitute an obstacle and a discontinuity. In this scenario, a nadiral flight with a standard overlapping (60\% along flight direction and 30\% along cross direction) is planned and then the three different approaches of estimation of the precisions are applied.

The model of classical photogrammetry (Kraus method) and the model of terrestrial close-range photogrammetry (Fraser method) depend on the shooting geometry represented by the distance between consecutive images (baseline B) and the principal distance (focal length c). The first one depends on the distance from the sensor to the object $(Z)$, while the second includes the mean number of images containing the object point (n) and a parameter called "geometric factor" (q).

Figure 2 shows how the classical photogrammetric approach is characterized by a relevant underestimation of expected precisions $(\sigma)$ and an estimation depending mostly on the relative flight quota (with uniform values of $4 \mathrm{~cm}$ on the beach). This is mainly due to different geometric settings between Kraus theory and UAS survey. In fact, Kraus formula is referred to a couple of images, whereas UAS survey generally uses a higher overlapping of images and a geometric configuration which is far from the "normal case" hypothesized by Kraus. If n, i.e. the effective number of the images that contain the object point, is not considered, the standard deviation increases.

The Fraser approach highlights an expected precision estimation which takes account of the real coverage of the images, therefore also of the morphology of the terrain. In Figure 2 relative to the close-range approach, the number of observation also influences the achievable precision (with values between $1.5 \mathrm{~cm}$ and $3 \mathrm{~cm}$ ); nevertheless, it strongly depends on the socalled "geometric factor" (q), difficult to suppose a priori. These analysis have stressed the need of a more rigorous approach, in order to calibrate the "geometric factor" of more expeditious models.

The rigorous approach, based on the network simulation, depends on the collimation expected precision but also on the 3D space network that involves each single object pixel and the corresponding images. The rigorous approach takes into consideration the geometric variations of the positioning of the cameras and the complexity of the shapes of the surveyed terrain. In fact, Figure 2 relative to the rigorous approach shows a greater variation of expected precisions $(\sigma)$ depending on the covering of the images.

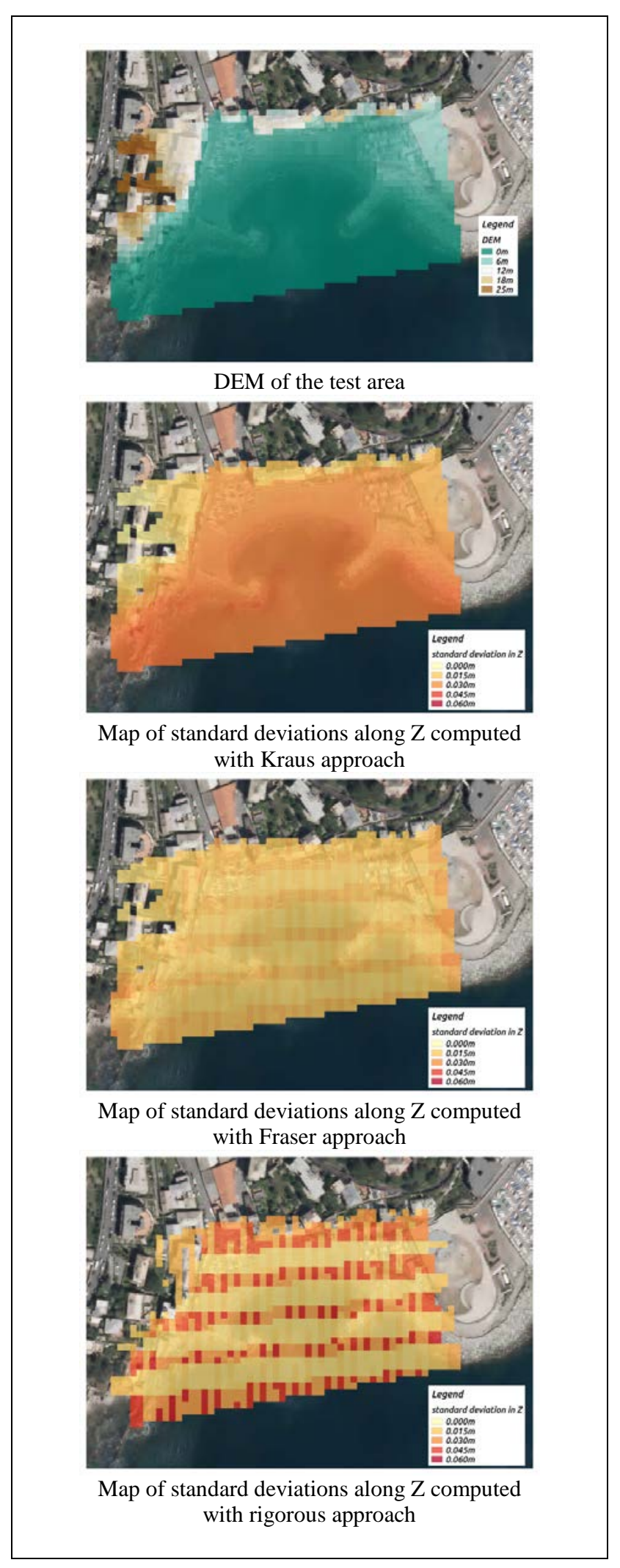

Figure 2. Maps of standard deviations along Z computed with different approaches 


\subsection{The proposed approach}

The example encourages the use of the rigorous approach for the estimation of the precisions. However, the rigorous approach shows a high computational difficulty, due to the large dimensions of the least squares adjustment system, which obviously depends on the spatial resolution of the DSM. Keeping a ground resolution of about one meter, and considering average extensions of 1-2 hectares, the computational time of the different solutions resulted compatible with the entire planning phase of a UAS flight.

For this reason, the rigorous solution is compared to the closerange solution computed fixing the geometric factor q equal to 1. The median of the ratio between each pixel on the ground of the two solutions provides a very accurate estimate of the geometric factor q. The efficiency of this estimate is highlighted by the standard deviation of the considered sample, which shows values of an order of magnitude lower than the magnitude of $q$.

In a planning of nadiral flight with a standard overlapping equal to $60 \%$ (like Vernazzola beach), Fraser suggests a value of the geometric factor $\mathrm{q}$ equal to 3.5. The rigorous approach estimated a value of 3.22 , with a standard deviation of 0.53 . Need moreover say that the precision of the measurements on the images $\left(\sigma_{p_{\xi}}\right)$ is fixed equal to one pixel and the a posteriori sigma $0\left(\hat{\sigma}_{0}\right)$ is fixed equal to $1 / 3$ of GSD (that is $3 \mathrm{~mm}$ ). At the state of the art, comparisons are made only on the $\mathrm{z}$ component, with the intention of implementing the forecast estimate in the other planimetric components in the future. Then, during the estimation procedure, the rigorous solution is evaluated with a low-resolution DSM (one/two meters or even more) to compute the geometric factor $\mathrm{q}$ and then to use this value inside the close-range procedure, potentially deploying an high-resolution DSM (one/two centimeters) without losing calculation efficiency and estimation accuracy.

If we carefully observe the formula (2) we can see that, $d, c, n$ are quantities observed while $\mathrm{q}$ and $\sigma_{\mathrm{p}_{\mathrm{x}}}$ are hypothesized parametric quantities. In the present work a probable value of $\sigma_{p_{\mathrm{x}}}$ equal to 1 pixel has been hypothesized. But if the precision of the measurements on the images $\sigma_{\mathrm{p}_{\mathrm{x}}}$ were greater or less, it influences the value calculated for $\mathrm{q}$. With the present rigorous method, the planning suite automatically evaluates the real ground coverage and the covering percentages for each image. At the end of the planning procedure, it is possible to export the georeferenced maps of precisions in .geotiff format, in order to use them in a Geographic Information System (GIS) environment.

\section{A CASE STUDY, SURVEYED BY NADIRAL AND OBLIQUE IMAGES}

The realistic planning has been applied to a case study, represented by the Castle of Casalbagliano, located in the countryside of Alessandria (Piedmont, Italy). The building is characterized by a square layout, typical of medieval fortifications. It was originally built using bricks, then restored several times; currently the building is abandoned and unsafe (Figure 3).

A survey has been performed in the test area and a complete dataset of nadiral and oblique images has been acquired by UAS. The Total Station (Leica Nova MS60 Multistation) and the GNSS have been used to georeference the Ground Control Points (GCPs). Eighteen GCPs have been materialized on the ground using black and white square targets (30 cm side) spread out over all the test area. GCPs survey is taken as reference to validate the reliability of the precisions which come from the planning suite.

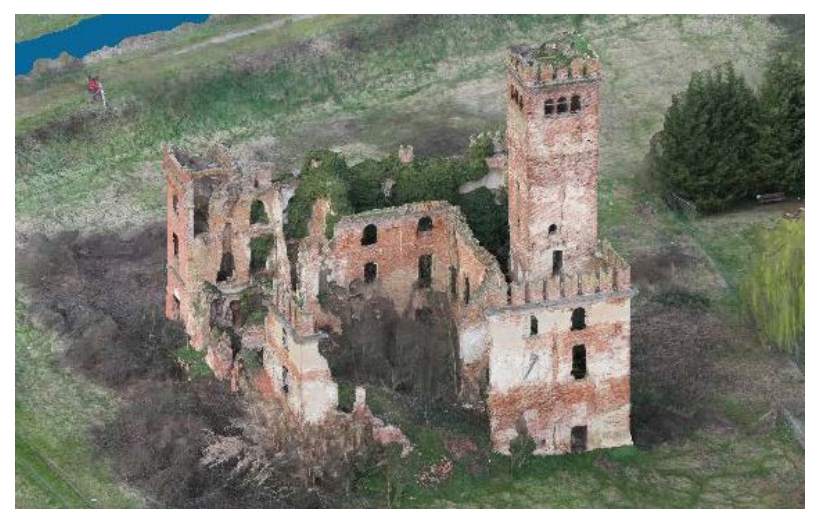

Figure 3. The castle of Casalbagliano in its 3D reconstruction

For the photogrammetric survey, the images have been acquired using a Canon EOS-M camera, with a fixed focal length equal to $22 \mathrm{~mm}$. The camera intrinsics, extrinsics, and lens distortion parameters were estimated with the Camera Calibrator app of Matlab ${ }^{\circledR}$. The camera has been put on a Mikrokopter Hexacopter, equipped with a gimbal stabilized platform. All the acquired images have been processed using the commercial software Agisoft Photoscan - version 1.2.6 (http://www.agisoft.com/), that provides the EO parameters, and performs the camera self-calibration (for both the considered dataset). In fact, in case of blocks acquired from UAS, it is quite important to refine the IO parameters, eventually changed after the estimation during a standard calibration procedure, because of the impacts suffered by the lens during take-off and landing. This software was chosen because the authors have a good experience of use and in the past years they have always found a remarkable reliability in the calculation of the aerial triangulation made in the orientation phase. The complete description of the survey campaign and of the elaboration settings is included in the paper by Gagliolo et al. (2017).

The EO parameters obtained with this AT are used as positions and attitudes of the projection centres in the estimation of a priori precisions. The resulting a priori precisions have been compared with the a posteriori accurate precision estimates, computed by means of Check Points (CPs), selected among the 18 measured points: among them, 6 were used as GCPs and 12 as CPs (Figure 4).

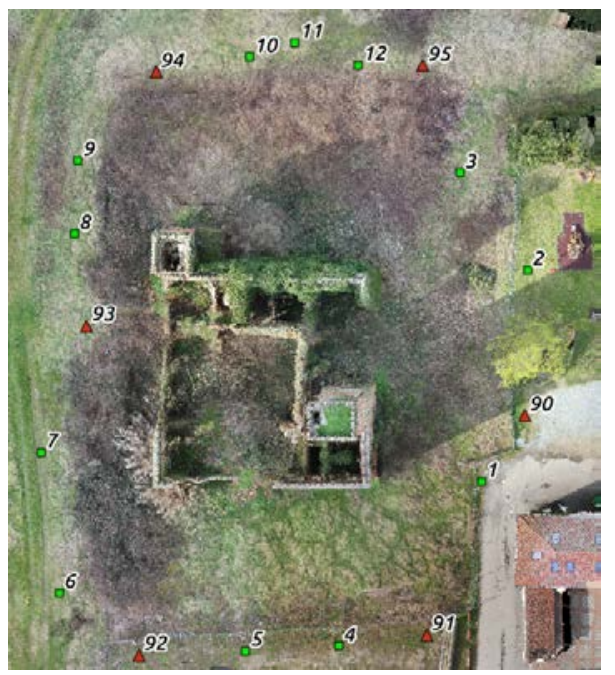


Figure 4. The position of GCPs and Check Points (CPs)

Two datasets of images, nadiral and oblique, were taken into consideration separately, in order to study the estimates of the precisions obtained depending on the two different geometric configurations.

The methodology explained in the previous paragraphs is applied, estimating the geometric factor $\mathrm{q}$ by the rigorous method with a low-resolution DSM (five meters) and then generating the map of the expected accuracies using the closerange approach (with estimated q) and a medium/highresolution DSM (one meter).

A comparison is performed between the simulation and the real case, that involves a series of accidental errors that cannot be foreseen a priori (for example images not perfectly focused or rolling shutter effect). For this reason, it is interesting to verify if the expected accuracies, computed during the design phase, are comparable to the real rms. This could confirm that the model has been properly chosen and the calibration of the weighing matrix has been accurately computed during the simulation. It is also interesting to check if the simulation and the real case have the same critical zones, i.e. areas where the precision drastically decreases. In fact, the aim of a network simulation is to predict the magnitude of the achievable precisions on the one hand, and on the other hand to highlight the eventual weaknesses of the designed flight plan.

The identification of these weaknesses also provides a guidance on how to redesign the flight and where to place the GCPs in order to provide further constraints to the resolution of the photogrammetric problem.

\subsection{Nadiral dataset}

The average flight height for the dataset with nadiral geometry was approximately $40 \mathrm{~m}$. A high overlapping has been guaranteed $(80 \%$ along flight direction and $70 \%$ along cross direction). The resulting average GSD was about $9 \mathrm{~mm}$.

The nadiral flight was designed and realised with 4 strips show in Figure 5.

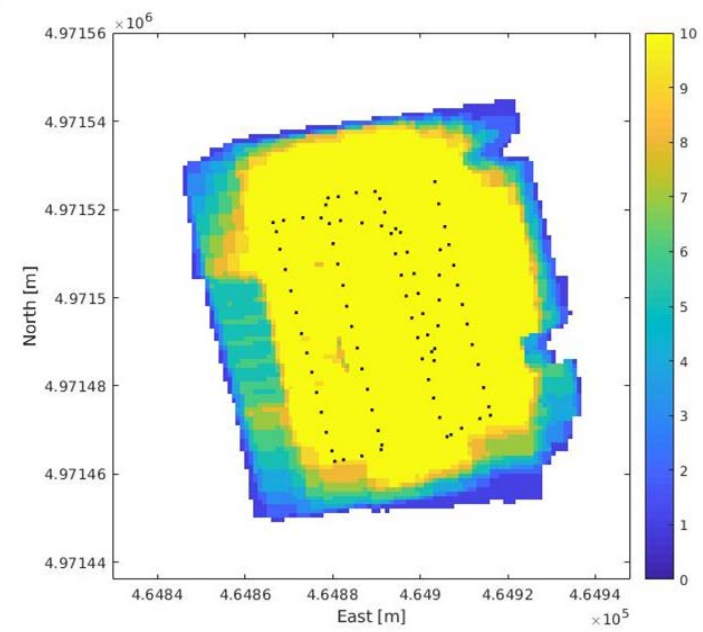

Figure 5. Camera location of the nadiral survey (black dots) and image overlapping (colour bar).

The software provides an estimate of the form coefficient $q$ equal to 5.74 . With this value of $q$ the map of the expected accuracies is generated using the close-range approach shown in Figure 6.

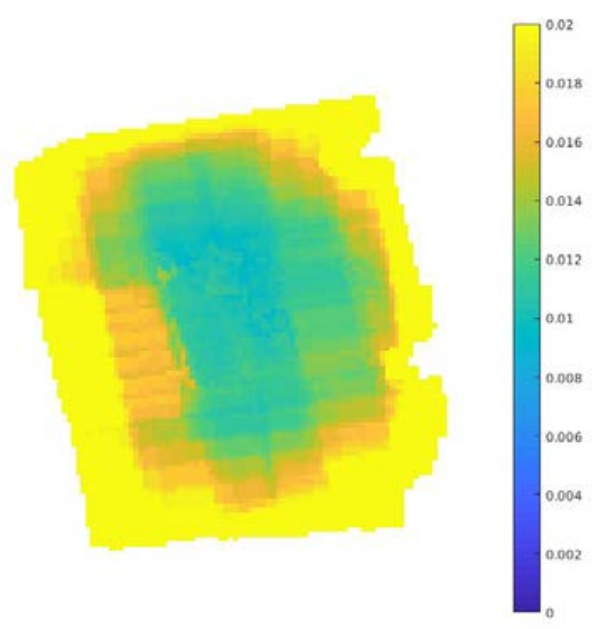

Figure 6. Map of the expected accuracies in $\mathrm{z}$ [m] for the nadiral survey.

As evident from Figure 6, the actual images overlapping show in Figure 5 and the morphology of the ground contribute to the determination of the accuracies. The difference between empirical precisions and theoretical precisions as shown in Figure 7, where in blue color are reported the empirical deviations and in red color are shown the expected theoretical precisions.

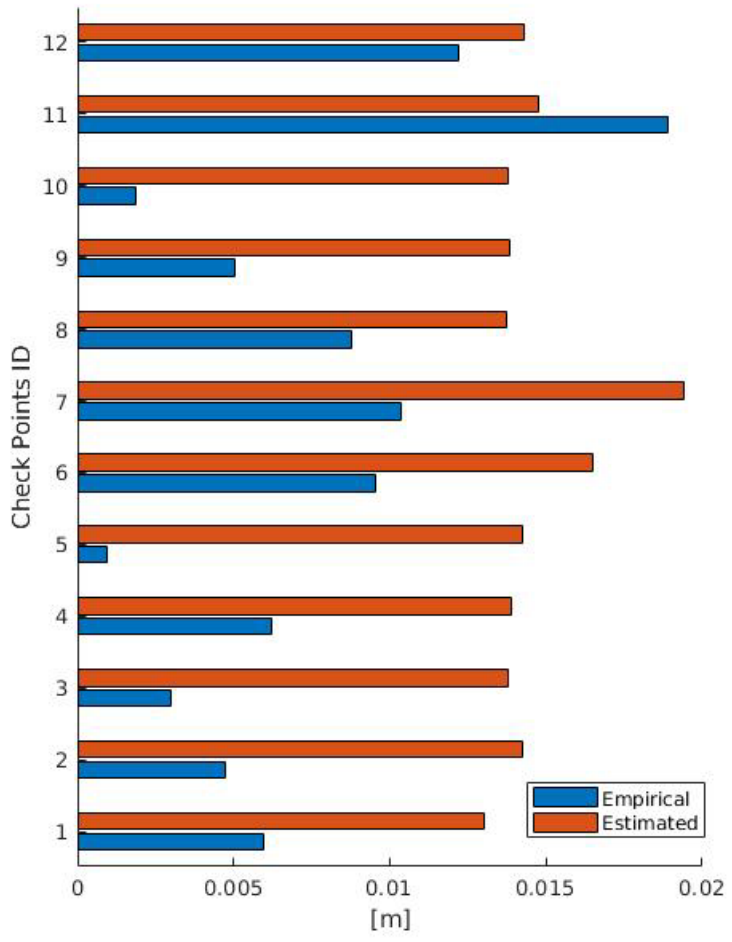

Figure 7. Empirical deviation and theoretical a priori rms estimated

The quantities shown are the deviations observed (empirical) and the estimates of the rms (estimated). The rms estimates are calculated only for the altimetry and refer to DSM with a resolution of $1 \mathrm{~m}$. The empirical value are the differences between the coordinates of the CPs measured with the Total Station and the values obtained as output of the Aerial Triangulation. It is necessary to underline that the quantities shown in the Figure 7 are conceptually different. At the same time the figure shows how the accuracy estimated a priori with 
our procedure is always greater than the observed one. Only the $\mathrm{CP}$ number 11 shows an observed deviations greater than the estimates of the rms, maybe due to accidental errors that cannot be foreseen a priori.

\subsection{Oblique dataset}

The flight with the oblique camera configuration has a flight height of about $50 \mathrm{~m}$ and describes a square along the building perimeter (maintaining a fixed distance of about $50 \mathrm{~m}$ ). The camera has been set up with a tilt angle of $45^{\circ}$, pointing at the centre of the described trajectory. The resulting overlapping was about $80 \%$. The oblique flight was designed and realised with 4 strips along the border of the survey zone show in Figure 8.

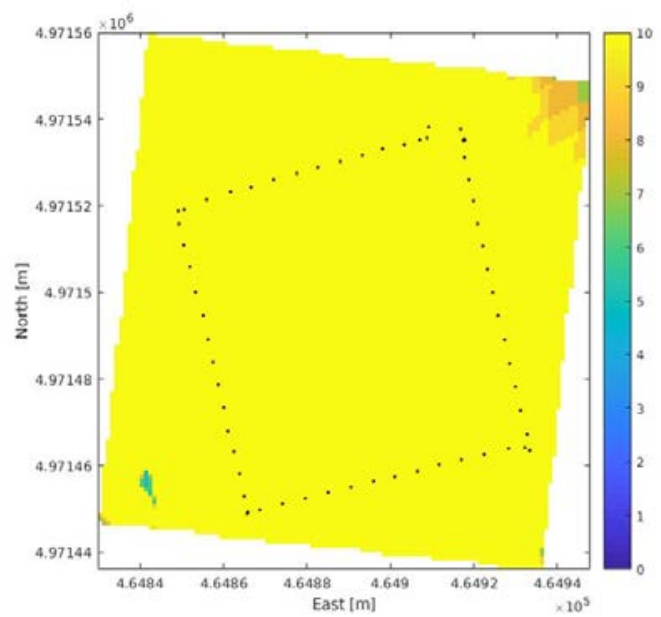

Figure 8. Camera location of the oblique survey (black dots) and image overlapping (colour bar).

Figure 8 shows also that flights with tilted camera generate more ground cover; ground points are therefore observed a greater number of times but with longer distances. Unfortunately, overlapping images does not take into account the presence of obstacles. It is the intention of the authors to implement this functionality soon. The GCPs and the CPs points are all external to the castle walls and therefore do not suffer from not having taken into account the obstructions.

The software provides an estimate of the form coefficient $q$ equal to 2.96 and a difference between empirical precisions and theoretical precisions as shown in Figure 9, where in blue color are reported the empirical deviations and in red color are shown the expected theoretical precisions. The quantities shown in Figure 9 are the deviations observed (empirical) and the estimates of the rms (estimated). Trend analysis shows that the empirical deviation are in some cases according to the trend of the accuracy estimated a priori with the presented procedure (for example CP3, CP10, CP11, CP12) but in some case an evident disagreement is observed (for example CP1, CP4, CP7, CP9). The causes of such discrepancies will need to be investigated in the future because. The preferred option is that the simulation and the real case have the same trend always i.e. highlight the same critical zones where the precision drastically decreases. The rms estimates (like in the nadiral case) are calculated only for the altimetry and refer to DSM with a resolution of $1 \mathrm{~m}$.

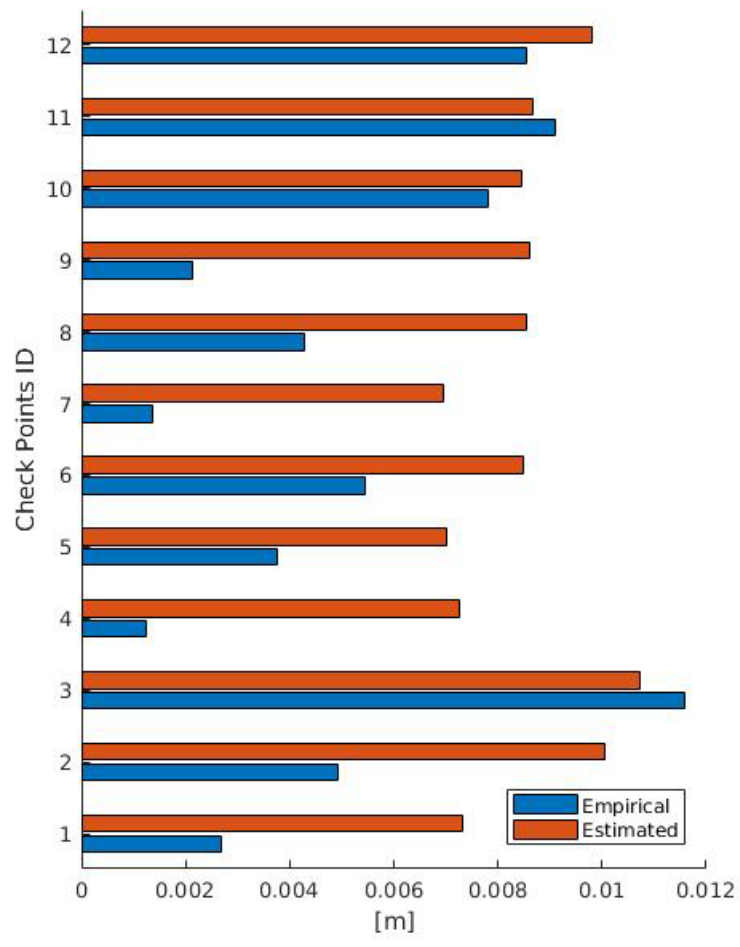

Figure 9. Empirical deviation and theoretical a priori rms estimated

\section{CONCLUDING REMARKS}

This work shows that the photogrammetric surveys from UAS can be very different from the traditional aerial surveys, if the high level of overlapping and the presence of oblique images are considered. These characteristics make UAS surveys very similar to close-range terrestrial surveys. However, if an accurate flight plan is desired, the application of the know rules for close-range photogrammetry to the aerial case is not sufficient, unless a new procedure to integrate both experiences is introduced. The conceived procedure, developed in the proposed "suite of tools", can provide adequate planning, analysis and management for a drone photogrammetric flight. In fact it is important to plan the photogrammetric survey predicting a realistic precisions that allow to view eventual dishomogeneity in the area. Meantime, when the survey is carried out, it is also important to check the overlapping and the precisions taking into account the effective morphology of the designed survey.

The developed procedure seems to show a valid way to plan even complex surveys in an easy way, still taking into account the complexity of the territory. The combination of the rigorous solution, which exploits a low-resolution DSM (one/two meters or even more) for computing the geometric factor q, with the close-range procedure (with the computed geometric factor) and a high-resolution DSM (one/two centimetres) is the solution that will be further developed in the near future. The proposed procedure is not simple, but it can result very useful in complex situations where high precision is required and meets the objective of highlighting critical areas of the survey that may not meet the required precisions. With this information the surveyor can decide to redesign the photogrammetric flight with different coverings or add GCPs in order to constrain the critical zones.

The obtained results encourage the authors to continue in this direction, in order to create an instrument for providing an 
accurate planning and for a priori predicting the resulting precisions.

\section{REFERENCES}

Fraser, C. S., 1984. Network design considerations for nontopographic photogrammetry. Photogrammetric Engineering and Remote Sensing, 50(8), pp. 1115-1126

Fraser, C. S., 1992. Photogrammetric measurement to one part in a million. Photogrammetric engineering and remote sensing, 58, pp. 305-310

Gagliolo, S., Fagandini, R., Federici, B., Ferrando, I., Passoni, D., Pagliari, D., Pinto, L., Sguerso, D., 2017. Use of UAS for the conservation of historical buildings in case of emergencies. International Archives of the Photogrammetry, Remote Sensing and Spatial Information Sciences, Vol. XLII-5/W1, p. 81-88.

Kraus, K., 1993. Photogrammetry, vol. 1, Fundamentals and standard processes. Dümmlers, Bonn

Pagliari, D., Rossi, L., Passoni, D., Pinto, L., De Michele, C., Avanzi, F., 2017. Measuring the volume of flushed sediments in a reservoir using multi-temporal images acquired with UAS. Geomatics, Natural Hazards and Risk, 8(1), pp. 150-166 